GRADIATION\&APPLICATIONS

ISSN 2466-4294 (online) | rad-journal.org

Vol. 2 | Issue 3 | pp. 186 - 191, 2017

doi: 10.21175/RadJ.2017.03.038

Original research paper

\title{
RADIATION RISK COMMUNICATION TO THE PATIENT*
}

\author{
Jozef Kubinyi' ${ }^{1}$,Jozef Sabol ${ }^{* *}$, Jana Hudzietzová ${ }^{3}$ \\ ${ }^{1}$ Institute of Nuclear Medicine, $1^{\text {st }}$ Faculty of Medicine, CU in Prague, Czech Republic \\ ${ }^{2}$ Faculty of the Security Management, PACR in Prague, Prague, Czech Republic \\ 3 Faculty of Biomedical Engineering, CTU in Prague, Prague, Czech Republic
}

\begin{abstract}
Ionizing radiation and radionuclides are widely used in diagnostic radiology, nuclear medicine and radiotherapy. Radiation related methods and procedures are especially useful in diagnostic applications where they provide valuable information about the patient conditions and problems. In this case, the effort is concentrated in obtaining the required diagnostic data while keeping the exposure to the patients to a very minimum level. On the other hand, the therapeutic use of radiation, in the form of external or internal exposure is aimed at delivering the relevant (rather high) doses to a particular volume in the organ or tissue in order to cure the tumour. In both of these modalities, the patients also receive a certain undesirable dose to healthy or normal tissues in the vicinity of the tumour. Obviously, any exposure may result in some stochastic effects characterized by a very small increase in the probability of developing additional cancers in years after the exposure. The paper discusses various methods of explaining the radiation risk to patients undergoing specific examinations or treatments involving radiation exposure. It also outlines the approach of the European Union and the situation in the Czech Republic.
\end{abstract}

Key words: Radiation risk, medical examination, patient, diagnoses, radiotherapy, stochastic effects, EU

\section{INTRODUCTION}

There is a variety of sources of ionizing radiation, such as X-ray based machines, radio-pharmaceuticals and also particle accelerators, which are used frequently in hospitals and clinics to perform diagnostic imaging examinations, medical interventions and special treatments aimed at curing some diseases, in particular cancer. Diagnostic procedures are important for the accurate diagnosis of diseases and injuries. Physicians and other medical personnel performing these operations are trained to use the minimum amount of radiation necessary for the technique applied. The benefits from these medical procedures greatly outweigh the potential small risk of harm due to an unwanted small exposure received by patients.

The increase in radiation related examinations in developed countries is becoming alarming. In Figure 1 [1], the situation in the USA regarding the increase in medical exposure during recent decades is illustrated. The exposure is mainly due to diagnostic radiology examinations and interventional radiology procedures. Diagnostic radiology includes the analysis of images obtained using X-rays, such as in plain radiography (e.g. chest or dental X-rays), fluoroscopy (e.g. with barium meal or enema) and computer tomography
(CT). Imaging modalities also comprise nuclear medicine examinations based on the use of unsealed radioactive sources (radio-pharmaceuticals) where the highest exposure to patients come primarily from SPECT/CT and PET/CT examinations. The other medical modality characterized by a high exposure is related to interventional radiology using minimally invasive image-guided procedures to diagnose and treat diseases (e.g. for guiding a catheter in a blood vessel).

The important components of all procedures involving radiation-related examinations or therapy have to be carefully scrutinized from the point of view of patients for a specific examination and informing. The patients should be informed about the potential risks related to the radiation exposure involved in simple language. However, one has to take into account that the risk of not making the diagnosis may be greater than the radiation risk.

Responding to patient concerns and questions about radiation risks can be one of the most challenging duties facing physicians, physicists and technicians. Patients often arrive with preconceived notions of risk based on misinformation they have seen in the media or read on the internet.

In any case, the patient should receive basic information about the specific use of radiation, where it is important to make patients aware of the radiation

\footnotetext{
* The paper was presented at the Fifth International Conference on Radiation and Applications in Various Fields of Research (RAD 2017), Budva, Montenegro, 2017.

sabol@polac.cz
} 
risk realistically in relation to other known risks. The patient should be able to distinguish between the medical procedures involving ionizing radiation from those relying on non-ionizing radiation imaging modalities relying on ultrasound or magnetic resonance techniques.

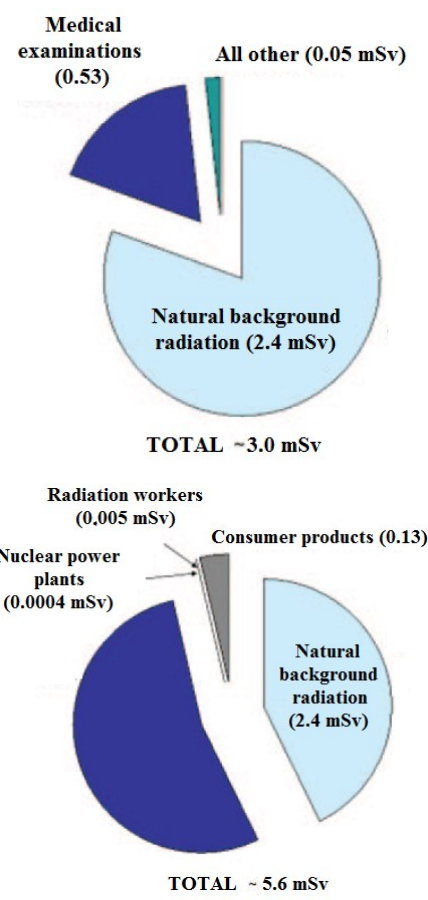

Figure 1. The US annual per capita effective dose from various sources in $1980(3 \mathrm{mSv})$ and in $2006(5.6 \mathrm{mSv})$. This trend can be soon expected in many other countries.

\section{STOCHASTIC AND DETERMINISTIC RADIATION EFFECTS}

In discussing the risk associated with the possible harmful effects of radiation exposure, a patient should be aware of the difference between stochastic effects, which are mainly attributed to normal diagnostic procedures, and deterministic effects, which are only relevant to the high radiation exposure encountered in therapy and in the case of accidents or radiological terrorist attacks $[2,3]$.

The risk of effects from diagnostic examinations is so low that it is more than compensated by the patient benefit from the use of radiation. The occurrence of stochastic effects is proportional to the effective dose received by a person. Just for comparison, an effective dose of $1 \mathrm{mSv}$ is associated with a risk of about $5 \times 10^{-5} \mathrm{mSv}^{-1}$, i.e. five in 100,000 persons exposed [4]. One chest X-ray examination (about $0.02 \mathrm{mSv}$ ) corresponds just to the exposure received by passengers during a five hour flight (due to higher contribution from cosmic radiation). These, and as well as other similar examples, should be used to inform patients about the risk of the exposure associated with the use of radiation in fully justified medical applications.
It may be impossible to even demonstrate that additional cancers have occurred as the normal incident rate of cancer deaths is about 20 percent plus or minus some natural variation in this rate. Diagnostic medical radiation exposures may increase this risk slightly, on the order of a fraction of percent. For an individual, this presents a very small risk but when there are many thousands of persons exposed to even that small radiation dose, there will be some (not negligible) additional number of cancers among them, which is undesirable from the societal point of view. Thus, even low exposures have to be under control.

Basically, stochastic effects are related to such cases when exposed and surviving cells may become modified by induced mutations (somatic, hereditary). These modifications may lead to two clinically significant effects: malignant neoplasms (cancer) and hereditary mutations.

On the other hand, the previously called deterministic effects, now referred to as tissue reactions, can only be observed after large absorbed doses of radiation above a certain threshold level and they are mainly a consequence of radiation induced cellular death. They occur only if a large proportion of cells in an irradiated tissue have been killed by radiation, and the loss cannot be compensated by increased cellular proliferation. Clinical examples of such effects are necrotic changes in skin, necrosis and fibrotic changes in internal organs, acute radiation sickness after whole body irradiation, cataracts, and sterility. The probability of deterministic effects does not vary with dose. Instead, there is a certain threshold dose for each specific harm below which the effect will not appear.

In addition, it has recently been observed that the possible effects may also include cataracts and cardiovascular outcomes at lower doses than previously considered [5-7].

Doses required to produce deterministic changes are in most cases large (usually in excess of 1-2 Gy) as illustrated in Figure 2. The specific response to deterministic effects may differ from person to persons.

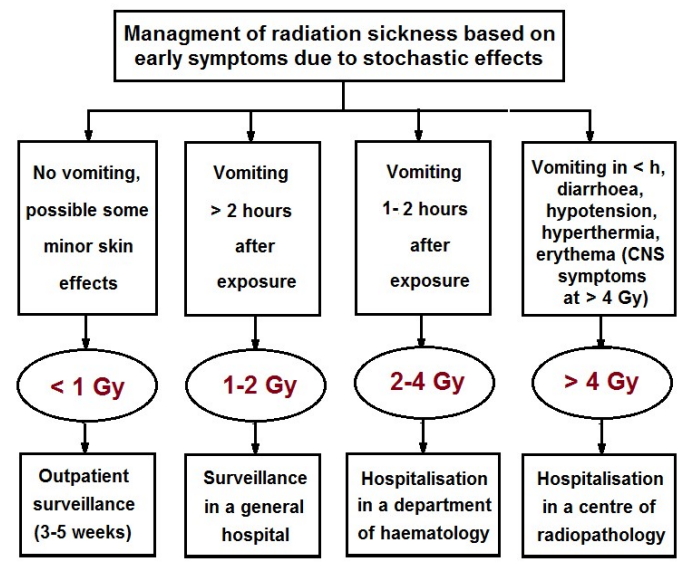

Figure 2. Characteristics of the impact and response to deterministic effects (CNS - Central Nervous System) 
Some of the deterministic effects occur in a small proportion of patients as side effects of radiotherapy. They can also be found after complex interventional investigations (such as vascular stenting) when long fluoroscopy times have been used. The essential feature of the dose-response relationship is the presence of a threshold dose. Below this dose no effect may be diagnosed but with increasing dose the intensity of the induced damage increases markedly, in some situations, dramatically.

\section{THE EXPOSURE TO PATIENTS FROM MEDICAL EXAMINATIONS}

In the past 100 years or so, diagnostic radiology, nuclear medicine and radiation therapy have evolved from the original rather primitive practices to advanced and efficient medical modalities which became an essential and indispensable tool for many branches and specialties of present medicine. Although the individual risk to a patient undergoing medical diagnostic examination is very low and, in most cases, comparable with the risk in everyday life, the impact of too many patients exposed too frequently may result in considerable consequences with respect to the total collective population exposure. This may result in such an increase in the number of additional cancer cases induced in a large population group that it cannot be neglected. The availability of radiation related facilities and, to a certain extent, the effort of some hospitals and clinics to increase the frequency of examinations due to the financial stimulation, contributes to the unnecessary high number of some kinds of examinations. Another reason behind this situation is the so-called "defensive medicine", when physicians, to be on the safe side, opt for some examinations which may not always be fully justified. When the CT modality first appeared, it was considered to be a magical apparatus; therefore, there were even cases when patients themselves insisted on being examined using that high-technology miracle.

Medical radiation can be delivered to the patient from a radiation source outside the patient (e.g. from an x-ray machine for diagnosis or a linear accelerator for radiotherapy). Regardless of the size of the dose the patients receive, they do not become radioactive or emit radiation. As a result, they present absolutely no radiation hazard to family or others.

The other way that medical radiation is given is by placing radioactive materials (radio-pharmaceuticals) in the patient. In these cases the patient becomes virtually a radioactive source and will emit radiation. For diagnostic nuclear medicine studies (such as a bone or thyroid scan), the amount of activity of radiopharmaceuticals injected is small and such patients present no hazard to their family or to the public. These patients are discharged immediately after the procedure.

Patients may undergo radiation therapy by having radioactivity injected or radioactive sources implanted in the tumour. Such patients may or may not present a hazard to others based upon the penetration capability of the radiation emitted by the radionuclide. Some are very poorly penetrating (such as iodine-125 prostate implants). Such patents are discharged. Others who receive iridium-192 or cesium implants must remain in the hospital until the sources are removed. The radiation is penetrating enough that visitors will be restricted from visiting the patient.

Patients treated with a high activity of ${ }^{131} \mathrm{I}$ for cancer of the thyroid, in some cases for hyperthyroidism, or patients with permanent implants of radioactive sources (special category of brachytherapy), once released home from a clinic or hospital may present some - however slight - risk to their family members if they do not observe specific rules of behaviour in such situations. These patients must be orally informed to avoid a close body contact with children and of other necessary precautions by specialists responsible for the conduct of their therapy.

On the basis of experimental clinical and epidemiological evidence, the assessment is also given of the probability of which cancers and hereditary mutations may be induced by doses of various magnitudes, most likely without a threshold dose (below which no effect would obtain).

Every effort should be undertaken to minimize the doses and, therefore, the risk from diagnostic uses of radiation, and to ensure that accurate high therapy radiation doses are delivered in the right volume to where tumour resides. This objective may be reached by avoiding unnecessary (unjustified) examinations, and optimizing the applied procedures both from the standpoint of diagnostic quality and of the reduction of excessive doses to patients. The optimization of patient protection in radiotherapy must depend on maintaining sufficiently high doses to irradiated tumours, securing a high cure rate, while protecting the healthy tissues to the largest extent possible. Problems related to the special protection of the human embryo and foetus in the course of diagnostic and therapeutic uses of radiation require some more specific approach.

Since future cancer risk is highly age dependent and many radiation induced cancers have latency periods of 10 to 20 years, individuals over 60 have minimal cancer risks from radiation exposure, while children have a lifetime risk of $10-15 \%$ simply due to the length of time available for a cancer to develop. This also has to be taken into account when assessing the risk due to radiation exposure.

\section{COMMUNICATION OF RADIATION RISK TO PATIENTS}

Commonly the risk of not making the diagnosis is greater than the radiation risk. Therefore, medical personnel has also to be trained how to transfer this information to their patients. A special situation may occur regarding the use of medical imaging in children. The medical community has to be well-positioned to address even such questions the parents and other caregivers may ask in a variety of scenarios "Could it hurt?"

There are many approaches to communicate medical radiation risks to patients, and each may have some advantages and disadvantages. These methods may be categorized into several categories which include the paternalistic approach, risk comparisons 
only approach, risk numerology approach, and the quality assurance approach [8].

The paternalistic approach was mainly applied in an earlier era, when the physician advised the patient what procedures and treatments were recommended, and the patient was expected to unquestionably follow such advice. Such an approach can be summarized as the "your doctor knows what you need best" method. Most patients usually agree that the radiation risk is acceptable when their physician recommends an exam and they believe the benefits from exams outweigh the risks associated with radiation.

Attempts of risk comparisons only approach to overcome the public's total lack of familiarity with radiation have included risk comparisons using the concept of effective dose to provide a relative risk of radiation exposure. Several comparison approaches are used, including comparisons with the background equivalent radiation time, comparisons with the number of airplane trips; expressing the dose as some multiple of a common examination, such as a chest radiograph; and by comparing an examination's dose to the safety levels prescribed for occupational exposures. Table 1 gives examples of such approaches for a chest radiograph and a CT scan of the abdomen (based on [9]).

Table 1. One of possible ways of comparing the risks from two common radiodiagnostic examinations with other risks

\begin{tabular}{|c|c|c|}
\hline $\begin{array}{l}\text { Comparison of typical } \\
\text { radiation exposure }\end{array}$ & $\begin{array}{l}\text { Chest } \\
\text { radiograph } \\
(\sim 0.1 \mathrm{mSv})\end{array}$ & $\begin{array}{l}\text { CT scan of the } \\
\text { abdomen } \\
(\sim 10 \mathrm{mSv})\end{array}$ \\
\hline $\begin{array}{l}\text { Equivalent to how many chest } \\
\text { radiographs }\end{array}$ & 1 & 100 \\
\hline $\begin{array}{l}\text { Background equivalent } \\
\text { radiation time Czech Republic } \\
(3 \mathrm{mSv} / \mathrm{y})\end{array}$ & $12 \mathrm{~d}$ & $3 \cdot 3 \mathrm{y}$ \\
\hline $\begin{array}{l}\text { No. of flights Prague - New } \\
\text { York }\end{array}$ & 2 & 230 \\
\hline $\begin{array}{l}\text { Fraction of occupational dose } \\
\text { limit }(20 \mathrm{mSv} / \mathrm{y})\end{array}$ & 0.0005 & 0.5 \\
\hline
\end{tabular}

The risk numerology approach is based on "informed consent" forms that include a fair amount of what can be termed "risk numerology" (e.g., relative risks, excess cancer rates, increased rates over background levels, and other hazard comparisons). Many investigators have used a factor for the increased risk of cancer in terms of probability of developing cancer related to the unit effective dose. However, even highly educated patients sometimes find it difficult to understand basic probability concepts or to perform even simple mathematic operations, such as comparing the magnitudes of two probabilities. For example, a probability for the additional risk of 1 in 20,000, corresponding to the risk associated with the exposure of $1 \mathrm{mSv}$, on a background risk of 3 in 10 (cancer rate in general population initiated by non- radiation initiative) is difficult, if not impossible, to grasp.

On some occasions, a visual aid may be very helpful in improving the dialogue about such risks, especially those based on dose proportions (ratio to a reference), what can be termed as a sort of a "Richter Scale of Radiation Risk". This scale may facilitate the comprehension of the risk by less knowledgeable persons and help them to distinguish between events of a wide range from practically no consequences via observable effects to severe consequences [10].

When discussing radiation risks, these should be described using broad categories such as those shown in Table 2 (e.g., negligible, < $0.1 \mathrm{mSv}$; minimal, 0.1-1 mSv; and minor, 1-10 $\mathrm{mSv}$ ).

Table 2. Examples of three types of imaging modalities and their equivalence to the exposure and risks (based on [8])

\begin{tabular}{lcccc}
\hline \multicolumn{1}{c}{ Imaging } \\
example & $\begin{array}{c}\text { Effect } \\
\text { dose } \\
\text { range } \\
\text { (mSv) }\end{array}$ & $\begin{array}{c}\text { Background } \\
\text { equivalent } \\
\text { radiation } \\
\text { time }\end{array}$ & Risk & $\begin{array}{c}\text { Probability } \\
\text { of cancer } \\
(\%)\end{array}$ \\
\hline $\begin{array}{l}\text { CT scan of } \\
\text { NM scan }\end{array}$ & $1-10$ & Years & Minor & $\sim 0.05$ \\
$\begin{array}{l}\text { Abdominal } \\
\text { radiograph }\end{array}$ & $0.1-1$ & Months & Minimal & $\sim 0.005$ \\
$\begin{array}{l}\text { Chest } \\
\text { radiograph }\end{array}$ & $<0.1$ & $\begin{array}{c}\text { Days to } \\
\text { weeks }\end{array}$ & Negligible & $\sim 0.0005$ \\
\hline
\end{tabular}

Quality assurances approach is used by some institutions which rely on what can be called "quality assurances" while communicating the benefit and risk in medical radiation. Quality assurance statements may include declarations claiming that the hospital or clinic uses the newest state-of-art imaging models or that their machines have been tested in accordance with the latest international standards. They may assure that their techniques were designed to deliver doses as low as reasonably achievable so patients do not really need to worry about any excessive exposure. Although these statements may indeed be true and may represent a healthy continuous improvement culture in the institution, they do not address benefits or risks in a quantifiable manner. In some cases, however, they may be reassuring.

\section{EUROPEAN UNION DIMENSION}

The European Union (EU) has addressed the safety and protection of patients against the unnecessary and unjustified radiation exposure in several of its documents. The main requirements, which are obligatory for the Member States were included in the relevant Directive [11], which emphasises the need for the justification of medical exposure, including strengthening the requirements concerning information to be provided to patients, the recording and reporting of doses from medical procedures, the use of diagnostic reference levels and the availability of dose-indicating devices.

The EU document has also highlighted the clinical responsibility of a practitioner for individual medical exposures, in particular, justification, optimisation, clinical evaluation of the outcome; cooperation with other specialists and staff, as appropriate, regarding the practical aspects of medical radiological procedures, obtaining information, if appropriate, on previous examinations, providing existing medical radiological information and/or records to other practitioners and/or the referrer, as required, and giving information on the risk of ionising radiation to patients and other individuals involved, as appropriate. 
The EU requires Member States to take appropriate steps in order to ensure that in the case of a patient undergoing diagnosis or treatment with radionuclides, the practitioner or other relevant assigned personnel provide the patient or their representative with sufficient information on the risks of ionizing radiation and appropriate instructions with a view to restricting doses to persons in contact with the patient as far as reasonably achievable.

\section{SitUATION IN THE CZECH REPUBLIC}

The basic legislation related the relationship between a patient and his/her medical doctor is now contained primarily in Act no. 372/2011 Coll., Health Services Act (HSA) [12], as amended which has been in effect since 1 April 2012. This Act postulates that health services can be provided to the patients only with their free and informed consent, unless the HSA Act provides otherwise.

Pursuant to this Act the patient is entitled to decide to which persons and to what scope the hospital is authorized to provide information about his/her health conditions and necessary medical procedures.

In accordance with the HSA no person shall interfere with the integrity of another person without his consent granted with the knowledge of the nature of the intervention and its possible consequences. In addition, every action in the context of health care requires the consent of the patient, unless the law provides that consent is not necessary. The informed consent to the provision of health services is, therefore, a legal act by a patient, which confers a particular patient medical service. Providers agree to give specific health services and, if necessary, information about specific interference to the patient's bodily integrity related to the provision of health services.

The informed consent for specific radio-diagnostic and nuclear medicine examinations, as well as for the therapy treatment based on ionizing radiation exposure takes into account the potential nature of the risk associated with such procedures.

This consent usually includes a completed questionnaire where the patient gives information about his/her health conditions, history of radiation related examinations and treatments, and previous allergic reactions. The female patients are supposed to provide information about their confirmed or potential pregnancy. In addition, patients are supposed to disclose the type of medication they were using in the past or they use at present.

The patient is expected to sign the informed consent where he/she express his/her acceptance of the examination or treatment proposed and that the procedures/risks have been explained to him/her by the relevant medical doctor. In this consent, the patient explicitly confirms that he/she

- Has been instructed and of their right to ask questions and decide freely whether he/she agrees with the proposed health service or health performance would agree or not,

- Has been informed in detail on the planned health performance,
- Has been given the reasons for the medical procedure and its benefits for his/her health conditions,

- Has been informed also about other possibilities and alternatives provided medical services and procedures, their appropriateness, benefits and risks,

- Has been informed of the possible risks and complications, the all this was told intelligibly to enable him/her to consider all possible impacts,

- Has had the opportunity to ask about everything thought to be important.

- Has declared that the information submitted was understood and there were no further questions,

- Has been instructed about the details of the proposed medical procedures including, if appropriate, alternative options using other equivalent modalities,

- Has hereby given his informed consent to the aforementioned health service and performance.

The patient information usually receives the information about the level of exposure associated with the examination or treatment including the explanation about potential risks resulted from the exposure. Here the radiologist is supposed to give an example of the situation with a similar risk as the risk from the prescribed radiation procedures.

\section{CONCLUSION}

Nowadays, many patients are more aware of the potential harm of ionizing radiation and ask questions which physicians have to be in a position to answer in such a way that patients would realistically perceive the magnitude of risk in the light of great benefit associated with radiation related examinations or treatment. This does not mean that they should be satisfied with the existing situation which, regarding the radiation risk communication to the patients, is still unsatisfactory at many hospitals and clinics. It would be desirable to pay more attention to training and educating non-radiation and referral physicians in understanding the radiation risk in perspective in order to be able to explain it to their patients.

It has been observed that non-radiologist physicians are often relatively uninformed about the involvement of radiation in diagnostic examinations. This includes specifically the dose and, therefore, the risk patients may be exposed to. Another problem may be attributed to understanding the published data regarding radiation exposure which is often confusing. Moreover, referring physicians do not always know the type of equipment being used at referring hospitals or clinics.

Ionising radiation is, therefore, one of the basic tools of contemporary medicine, both in diagnosis and therapy. Practice of contemporary, advanced medicine, without use of ionizing radiation appears currently unthinkable. 


\section{REFERENCES}

1. F. Mettler et al., "Radiologic and nuclear medicine studies in the United States and worldwide," Radiology, vol. 253 , no. 2, pp. 520 - 531, Nov. 2009.

DOI: $10.1148 /$ radiol.2532082010 PMid: 19789227

2. J. Sabol and B. Šesták, "Communication with the public in radiation protection and nuclear safety and security," in Proc. European Nuclear Conference - ENC 2014, pp. 150 - 157, Marseille, France, 2014. Retrieved from:

http://www.euronuclear.org/events/enc/enc2014/tran sactions/ENC2014-transactions-reviewed.pdf Retrieved on: Aug. 3, 2017

3. J. Sabol et al, "Current Activities of the European Union in Fighting CBRN Terrorism Worldwide," in Nuclear threats and security challenges, S. Apikyan and D. Diamond Eds., Los Angeles (CA), USA: Springer Science, 2015, ch. 15, pp. 157- 167.

DOI: $10.1007 / 978-94-017-9894-5$

4. "The 2007 Recommendations of the International Commission on Radiological Protection", Ann. ICRP, vol. 37, no. 2-4, 2007.

Retrieved from:

http://www.icrp.org/docs/ICRP Publication 103Annals of the ICRP $37(2-4)$-Free extract.pdf Retrieved on: Aug. 3, 2017

5. F. A. Stewart et al., "ICRP Statement on tissue reactions/Early and late effects of radiation in normal tissues and organs - threshold doses for tissue reactions in a radiation protection context," Ann. ICRP, vol. 41, no. 1-2, 2012.

Retrieved from:

http://files.site-

fusion.co.uk/webfusion117640/file/icrp118 1.pdf

Retrieved on: Aug. 3, 2017

6. N. Hamada et al., "Emerging issues in radiogenic cataract and cardiovascular disease," J. Radiat. Res., vol. 55, no. 5, pp. $831-846$, Sep. 2014.

DOI: $10.1093 / \mathrm{jrr} / \mathrm{rru036}$

PMid: 24824673

PMCid: PMC4202294
7. K. Kamiya et al., "Long-term effects of radiation exposure on health," Lancent, vol. 386, no. 9992, pp. $469-478$, Aug. 2015. DOI: $10.1016 /$ So140-6736(15)61167-9

8. T. Lawrence et al., "Fears, feelings, and facts: Interactively communicating benefits and risks of medical radiation with patients," $A J R \quad A m$. J. Roentgenol., vol. 196, no. 4, Apr. 2011. DOI: 10.2214/AJR.10.5956

9. "Radiation dose management for fluoroscopically guided interventional medical procedures," NCRP, Bethesda (MD), USA, Rep. 168, 2010.

10. K. Mück et al., "A proposal for radiation protection scale to better communicate with the public," in Proc. IRPA-1O Int. Cong. Int. Rad. Prot. Assoc., Hiroshima, Japan, 2000.

Retrieved from:

https://pdfs.semanticscholar.org/f64b/e03a893f14f3c7 ae8c7de93f8b30b0499c9o.pdf

Retrieved on: Jul. 10, 2017

11. The Council of the European Union. (Dec. 5, 2013) Council Directive 2013/59/Euratom laying down basic safety standards for protection against the dangers arising from exposure to ionising radiation, and repealing Directives 89/618/Euratom, 90/641/Euratom, 96/29/Euratom, 97/43/Euratom and 2003/122/Euratom.

Retrieved from:

https://ec.europa.eu/energy/sites/ener/files/document s/CELEX-32013L0059-EN-TXT.pdf

Retrieved on: Jul. 23, 2017

12. The Parliament of the Czech Republic. (Nov. 6, 2011) Patient's consent with provision of information in agreement with the Act No. 372/2011 Coll., on health services and the terms and conditions for the providing of such services.

Retrieved from:

https://www.dentmedico.cz/wpcontent/uploads/2016/10/Pouceni-a-souhlas-pacientaAJ.pdf

Retrieved on: Jul. 23, 2017 